\title{
FINITE CODIMENSIONAL IDEALS IN BANACH ALGEBRAS
}

\author{
KRZYSZTOF JAROSZ
}

\begin{abstract}
Let $A$ be a commutative, selfadjoint, point spectral Banach algebra and let $M$ be a finite codimensional closed subspace of $A$ such that for each $f$ in $M$ there are $n$ distinct maximal ideals $I_{1}^{f}, \ldots, I_{n}^{f}$ of $A$ with $f \in I_{j}^{f}$. We prove that then there are distinct maximal ideals $I_{1}, \ldots, I_{n}$ of $A$ such that $M \subset I_{1} \cap \cdots \cap I_{n}$; in particular if $\operatorname{codim}(M)=n$, then $M$ is an ideal.
\end{abstract}

1. Introduction. In this note we investigate the following problem: Let $A$ be a commutative Banach algebra and let $M$ be a closed finite codimensional subspace of $A$. Let us assume that any element of $M$ is contained in at least $n$ different closed maximal ideals of $A$. Does it imply that there are $n$ distinct maximal ideals $I_{1}, \ldots, I_{n}$ of $A$ such that $M \subset I_{1} \cap \cdots \cap I_{n}$ ?

The source of this problem is the Gleason-Kahane-Żelazko theorem $[\mathbf{2}, \mathbf{4}]$ which states that the answer is positive if $A$ has a unit and if $\operatorname{codim}(M)=1=n$. This classical result has been extended for some selfadjoint algebras for $\operatorname{codim}(M)=$ $n>1$ by Warner and Whitley [6, 7]. In 1985, in [3] it has been proved that the answer is positive for $A=C(S), S$ a compact Hausdorff space, for $n=1$ and that the same holds for $n>1$ if and only if each finite subset of $S$ is $G_{\delta}$. In a very recent paper [5], N. V. Rao has generalized the above result to any selfadjoint, regular Banach algebras. In this paper we prove that this result can also be extended to selfadjoint, point spectral algebra as well as some other Banach algebras.

For more biographical information and examples concerning our problem see [3].

2. Definitions and notation. For a commutative Banach algebra $A$ we denote by $\mathfrak{M}(A)$ the maximal ideal space of $A$ equipped with the weak* topology. For $f$ in $A$, by $\hat{f}$ we denote the Gelfand transform of $f$. We put $\operatorname{re}(A)=\{f \in$ $A: \hat{f}$ is a real valued function $\}$ and we call $A$ selfadjoint if $A=\operatorname{re}(A)+i \operatorname{re}(A)$. For $f, g$ in $A$, by $f \mid g$ we mean that $f$ divides $g$; this means that there is an $h$ in $A$ such that $g=h f$. We call $A n$-point spectral, $n=0,1,2, \ldots$, if for each $n$-point set $K$ in $\mathfrak{M}(A)$ there is exactly one closed ideal $J$ such that $\{I \in \mathfrak{M}(A): J \subset I\}=K$. Hence by 0 -point spectral property we mean: if $J$ is a closed ideal in $A$ contained in no closed maximal ideal of $A$, then $J=A$.

We say that a commutative Banach algebra $A$ has the $P(k, n)$ property if the following holds: Let $M$ be a closed $k$ codimensional subspace of $A$ such that for any $f$ in $M$ there are at least $n$ distinct closed maximal ideals $I_{1}^{f}, \ldots, I_{n}^{f}$ containing $f$. Then there are $n$ distinct maximal ideals $I_{1}, \ldots, I_{n}$ of $A$ containing $M$.

Received by the editors December 11, 1985 and, in revised form, April 7, 1986.

1980 Mathematics Subject Classification (1985 Revision). Primary 46J05. 


\section{The result.}

THEOREM 1. For any commutative, selfadjoint Banach algebra $A$ we have:

(i) if there is an $f$ in $A$ such that the ideal $f A=\{f g: g \in A\}$ is dense in $A$, then $A$ has the $P(k, 1)$ property for any $k \in \mathbf{N}$;

(ii) if for any n-point subset $\left\{I_{1}, \ldots, I_{n}\right\}$ of $\mathfrak{M}(A)$ there is an $f$ in $A^{\prime}=I_{1} \cap \cdots \cap I_{n}$ such that $f A^{\prime}$ is a dense subset of $A^{\prime}$, then $A$ has the $P(k, n+1)$ property for any $k \in \mathbf{N}$.

There are a large number of Banach algebras which satisfy the assumptions of Theorem 1, for example $L^{1}(G)$ algebras. The following well-known proposition gives information about relations among the hypotheses of the theorem, the topological properties of $\mathfrak{M}(A)$, and the spectral properties of $A$.

PROPOSITION 1. For any commutative, selfadjoint Banach algebra $A$ we have:

(a) $\mathfrak{M}(A)$ is $\sigma$-compact if and only if there is an $f$ in $A$ such that

$$
\hat{f}>0 \text { on } \mathfrak{M}(A),
$$

(b) if $A$ is 0-point spectral, then for any $f$ in $A$ which satisfies (1), the ideal $f A$ is dense in $A$,

(c) $\mathfrak{M}(A)$ is $\sigma$-compact and each point of $\mathfrak{M}(A)$ is a $G_{\delta}$ set if and only if for any finite subset $K$ of $\mathfrak{M}(A)$ there is an $f$ in $A$ such that

$$
\hat{f} \geq 0 \quad \text { on } \mathfrak{M}(A) \quad \text { and } \quad \hat{f}^{-1}(0)=K,
$$

(d) if $A$ is n-point spectral, $K=\left\{I_{1}, \ldots, I_{n}\right\} \subset \mathfrak{M}(A)$, and if $f$ in $A$ satisfies (2), then the ideal $f A$ is dense in $I_{1} \cap \cdots \cap I_{n}$.

From Theorem 1 and the above proposition we get

THEOREM 2. Let $A$ be a selfadjoint, commutative Banach algebra and let $k$ be a positive integer. We have:

(i) if $A$ is 0 -point spectral, then $A$ has the $P(k, 1)$ property if and only if $\mathfrak{M}(A)$ is $\sigma$-compact,

(ii) if $A$ is n-point spectral, then $A$ has the $P(k, n+1)$ property if and only if $\mathfrak{M}(A)$ is $\sigma$-compact and each point of $\mathfrak{M}(A)$ is a $G_{\delta}$ set.

ProOF OF THEOREM 2. The "if" parts of both the above statements are immediate consequences of Theorem 1 and Proposition 1. The "only if" parts are easy and very general: For any commutative Banach algebra $A$ and for any $f$ in $A$ the set $f^{-1}(\mathbf{R}-\{0\})$ is $\sigma$-compact. Hence, assuming $\mathfrak{M}(A)$ is not $\sigma$-compact, we get that any $f$ from $A$ is contained in some, and in fact in infinitely many, maximal closed ideals of $A$, but evidently not all finite codimensional subspaces of $A$ are contained in some maximal ideal. Assume now that $I \in \mathfrak{M}(A)$ is not a $G_{\delta}$ set and let $M$ be a finite codimensional subspace of $I$. Any $f$ from $M$ is contained in $I$ so $\hat{f}$ has infinitely many zeros in $\mathfrak{M}(A)$, but $M$ need not be contained in more than this one maximal ideal of $A$.

To prove Theorem 1 we need the following two propositions, which are easy to verify. 
Proposition 2. Let $B$ be a Banach space and $E \subset A$ be subspaces of $B$ with $A$ dense in $B$. Then

$$
\operatorname{dim}(B / \bar{E})=\operatorname{dim}(A / E) .
$$

Proposition 3. Let $B$ be a Banach space, $M$ a finite codimensional closed subspace of $B$, and $A$ a dense subspace of $B$. Then

$$
\operatorname{dim}(B / M)=\operatorname{dim}(A / M \cap A) .
$$

ProOF OF THEOREM 1 . To prove (i) let $M$ be a closed, $k$-codimensional subspace of $A$ and assume that each element of $M$ is contained in some closed maximal ideal of $A$.

Assume $A$ has a unit. Put $\hat{M}=\{\hat{f}: f \in M\}$, and put $\mathcal{M}=\operatorname{cl}(M) \subset C(\mathfrak{M}(A))$ where in the sequel we denote by $\operatorname{cl}(\cdot)$ the closure in the sup norm topology. The set of noninvertible elements is sup-norm closed so, by Proposition 2 and Theorem 2 of [3], we get that there is an $x$ in $\mathfrak{M}(A)$ such that $\hat{M} \subset \mathcal{M} \subset \operatorname{ker} \delta_{x}$ and we are done.

Assume now $A$ has no unit. Let $\mathfrak{M}^{*}=\mathfrak{M} \cup\{*\}$ be a one-point compactification of $\mathfrak{M}=\mathfrak{M}(A)$. Notice that

$$
f(x) \rightarrow 0 \text { as } x \rightarrow * \text { for any } f \text { in } A .
$$

By our assumption there is an $f_{0}$ in $A$ such that $\hat{f}_{0}>0$ on $\mathfrak{M}(A)$ and $f_{0} A$ is dense in $A$.

Put

$$
\begin{gathered}
A_{0}=\left\{f+\alpha f_{0}: f \in A, \alpha \in \mathbf{C}, f_{0} \mid f\right\}, \\
A_{1}=\left\{f+\alpha f_{0}: f \in A, \alpha \in \mathbf{C}, f_{0}\left|f, \bar{f}_{0}\right| f\right\}, \\
A_{0}=\hat{A}_{0} / \hat{f}_{0}=\left\{\hat{f} / \hat{f}_{0} \in C\left(\mathfrak{M}^{*}\right): f \in A_{0}\right\}, \\
A_{1}=\hat{A}_{1} / \hat{f}_{0}, \quad M_{0}=A_{0} \cap M, \quad \mathcal{M}=\hat{M}_{0} / \hat{f}_{0} .
\end{gathered}
$$

It is easy to verify that $A_{1}$ is an algebra, is selfadjoint, contains the unit and separates points of $\mathfrak{M}^{*}$, and that $A_{1} \subset A_{0}$. Hence

$$
\operatorname{cl}\left(A_{1}\right)=\operatorname{cl}\left(A_{0}\right)=C\left(\mathfrak{M}^{*}\right)
$$

By our assumption $\bar{A}_{0}=A$, so by Propositions 3 and 2 we get

$$
k=\operatorname{dim}(A / M)=\operatorname{dim}\left(A_{0} / A_{0} \cap M\right)=\operatorname{dim}\left(A_{0} / \mathcal{M}\right)=\operatorname{dim}\left(C\left(\mathfrak{M}^{*}\right) / \operatorname{cl}(\mathcal{M})\right) .
$$

Hence, by Theorem 2 of [3], there is an $x$ in $\mathfrak{M}^{*}$ such that $\mathcal{M} \subset \operatorname{ker} \delta_{x}$. Assume that $x=*$. Then $M_{0} \subset f_{0} A \nsubseteq A_{0}$ and by our assumption $f_{0} A$ is dense in $A$, so by Proposition 3 we get

$$
\begin{aligned}
k & =\operatorname{dim}(A / M)=\operatorname{dim}\left(A_{0} / A_{0} \cap M\right) \\
& =1+\operatorname{dim}\left(f_{0} A / A_{0} \cap M\right)=1+\operatorname{dim}(A / M)=k+1 .
\end{aligned}
$$

This proves that $x \neq *$ and ends the proof of (i).

Now we prove (ii) by induction on $n$. Let $M$ be a closed subspace of $A$ of finite codimension and such that each element of $M$ is contained in at least $n+1$ distinct maximal closed ideals of $A$. Assume $A$ has the property described in (ii). Let $I_{1}, \ldots, I_{r}$ be all the maximal ideals which contain $M$ and assume that $r<n+1$. 
By our assumption there is an $f$ in $A^{\prime}=I_{1} \cap \cdots \cap I_{r}$ such that $f A^{\prime}$ is dense in $A^{\prime}$. We have $\mathfrak{M}\left(A^{\prime}\right)=\mathfrak{M}(A)-\left\{I_{1}, \ldots, I_{r}\right\}$ so we have the following situation: $M$ is a finite codimensional closed subspace of the algebra $A^{\prime}$ such that any element of $M$ is contained in at least $n+1-r$ maximal ideals of $A^{\prime}$. Hence by (i) there is a maximal closed ideal $I$ of $A^{\prime}$ such that $M \subset I$; this contradicts the assumption that $I_{1}, \ldots, I_{r}$ are all the maximal closed ideals which contain $M$ and ends the proof.

\section{REFERENCES}

1. C P. Chen and P. J. Cohen, Ideals of finite codimension in commutative Banach algebras, manuscript.

2. A. M. Gleason, A characterization of maximal ideals, J. Analyse Math. 19 (1967), 171-172.

3. K. Jarosz, Finite codimensional ideals in function algebras, Trans. Amer. Math. Soc. 287 (1985), 779-785.

4. J. P. Kahane and W. Żelazko, Characterization of maximal ideals in commutative Banach algebras, Studia Math. 29 (1968), 339-343.

5. N. V. Rao, Closed subspaces of finite codimension in regular selfadjoint Banach algebras, manuscript.

6. C. R. Warner and R. Whitley, A characterization of regular maximal ideals, Pacific J. Math. 30 (1969), 277-281.

7. 263-267.

INStitute of Mathematics, Warsaw University, P.K.I N. 9P. 00-901 WarsaW, POLAND

Current address: Department of Mathematics, University of California, Santa Barbara, California 93106 